\title{
On the importance of the equation of state for the neutrino-driven supernova explosion mechanism
}

\author{
Yudai Suwa \\ Yukawa Institute for Theoretical Physics, Kyoto University, Oiwake-cho, Kitashirakawa, \\ Sakyo-ku, Kyoto, 606-8502, Japan; email: suwa@yukawa.kyoto-u.ac.jp
}

\begin{abstract}
We present two-dimensional numerical simulations of core-collapse supernova including multi-energy neutrino radiative transfer. We aim to examine the influence of the equation of state (EOS) for the dense nuclear matter. We employ four sets of EOSs, namely, those by Lattimer and Swesty (LS) and Shen et al., which became standard EOSs in the core-collapse supernova community. We reconfirm that not every EOS produces an explosion in spherical symmetry, which is consistent with previous works. In two-dimensional simulations, we find that the structure of the accretion flow is significantly different between LS EOS and Shen EOS, inducing an even qualitatively different evolution of the shock wave, namely, the LS EOS leads to shock propagation beyond $2000 \mathrm{~km}$ from the center, while the Shen EOS shows only oscillations within $500 \mathrm{~km}$. The possible origins of the difference are discussed.
\end{abstract}

Keywords. equation of state - hydrodynamics - neutrinos - stars: neutron — supernovae: general

\section{Introduction}

Core-collapse supernova explosions are triggered by the gravitational energy released during the transition from a stellar core into a protoneutron star (PNS), which has a temperature on the order of tens of $\mathrm{MeV}$ and densities on the order of normal nuclear matter density $\left(3 \times 10^{14} \mathrm{~g} \mathrm{~cm}^{-3}\right)$. There are only few equations of state (EOS) valid for these conditions. The most commonly used nuclear EOS in supernova simulations are the EOS from Lattimer \& Swesty (1991) (hereafter LS), based on the incompressible liquiddrop model including surface effects, and from Shen et al. (1998) (SHEN). The latter is based on relativistic mean field (RMF) theory and Thomas-Fermi approximation.

There are several studies, which investigated EOS dependences on the hydrodynamical features in spherical symmetry, while a similar study in multi-dimensional simulation is not performed so far. Recently, several simulations that successfully produced the explosion are reported (Marek \& Janka (2009), Suwa et al. (2010)). Therefore, we can now investigate how the EOS could affect the supernova dynamics.

In this paper we present results of numerical simulation of core-collapse supernovae of massive iron-core progenitors. Our model is based on neutrino radiation hydrodynamics and includes multi-energy radiative transfer. We employ four EOS, LS (with three values of incompressibility) and SHEN. We focus on the shock formation and evolution on a long timescale (more than $500 \mathrm{~ms}$ after core bounce) and investigate whether the shock finally obtains enough energy via neutrino heating to expand outward.

\section{Numerical Method and Results}

Methods. Our 2D simulations are performed using a code which is based on the spectral neutrino transport scheme IDSA, developed by Liebendörfer et al. (2009), and the 


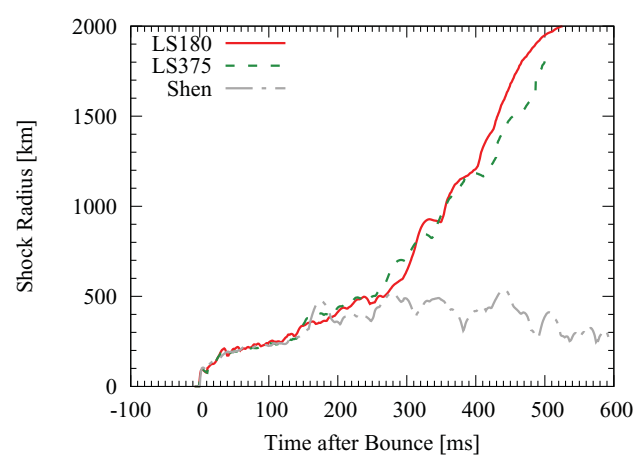

Figure 1. The time evolution of the maximum radius of shock wave.

ZEUS-2D code (Stone \& Norman (1992), Suwa et al. (2010), Suwa et al. (2011)). In this study, we employ the two standard EOS for matter in NSE, LS and SHEN, in supernova simulations. As for the LS, there are three different versions available, for three different values of the incompressibility, $K=180 \mathrm{MeV}$ (LS180), $220 \mathrm{MeV}$ (LS200), and $375 \mathrm{MeV}$ (LS375). Here we present results of LS180 and LS375 because LS220 is similar to LS180 in our simulation. We use the $15 \mathrm{M}_{\odot}$ progenitor from Woosley \& Weaver (1995).

Results. We compare the results obtained in core-collapse supernova simulations of the $15 \mathrm{M}_{\odot}$ progenitor using the different EOS, i.e., LS180, LS375, and SHEN. Fig. 1 shows the shock-radius evolution for each model. Each of them has three lines, corresponding to maximum, angular average and minimum shock radii (from top to bottom). From Fig. 1, we can see that LS180 and LS375 (red solid lines and green dotted lines) show similar trajectories. There is a gradual expanding phase for the postbounce time $t_{\mathrm{pb}} \lesssim 200$ $300 \mathrm{~ms}$ where the maximum shock radii reach about $500 \mathrm{~km}$, after which the shock radii start to grow more rapidly for both models. Later, no further contraction is observed. The maximum shock radii reach about $2000 \mathrm{~km}$ for LS180 and $1800 \mathrm{~km}$ for LS375 at $500 \mathrm{~ms}$ post bounce. On the other hand, for the 2D simulation using SHEN the shock wave does not continue to grow to increasingly larger radii, even at late times. Although conclusions about possible explosions using LS180 and LS375 are still weak at the post bounce times when the $2 \mathrm{D}$ simulations were stopped (the explosion energy remains $\sim 10^{50} \mathrm{erg}$ in both cases), explosions for the 2D simulations using SHEN are highly unlikely to occur.

Numerical computations were in part carried on Cray XT4 and medium-scale clusters at CfCA of the National Astronomical Observatory of Japan, and on SR16000 at YITP in Kyoto University. This study was supported in part by the Grants-in-Aid for the Scientific Research from the Ministry of Education, Science and Culture of Japan (Nos. 19540309, 20740150 and 23840023) and MEXT HPCI STRATEGIC PROGRAM.

\section{References}

Lattimer, J. M. \& Swesty, F. D. 1991, Nuclear Physics A, 535, 331

Liebendörfer, M., Whitehouse, S. C., \& Fischer, T. 2009, Astrophys. J., 698, 1174

Marek, A. \& Janka, H. 2009, Astrophys. J., 694, 664

Shen, H., Toki, H., Oyamatsu, K., \& Sumiyoshi, K. 1998, Nucl. Phys., A637, 435

Stone, J. M. \& Norman, M. L. 1992, Astrophys. J., 80, 753

Suwa, Y. et al. 2010, PASJ, 62, L49

Suwa, Y., Kotake, K., Takiwaki, T., Liebendörfer, M., \& Sato, K. 2011, Astrophys. J., 738, 165

Woosley, S. E. \& Weaver, T. A. 1995, Astrophys. J., 101, 181 